\title{
In vitro effect of a corrosive hostile ocular surface on candidate biomaterials for keratoprosthesis skirt
}

\author{
Xiao Wei Tan, ${ }^{1}$ Andri Riau, ${ }^{1}$ Zhi Long Shi, ${ }^{2}$ Anna C S Tan, ${ }^{3}$ Koon Gee Neoh, ${ }^{2}$ \\ Khiam Aik Khor, ${ }^{4}$ Roger W Beuerman, ${ }^{1,5,6}$ Donald Tan, ${ }^{2,3}$ Jodhbir S Mehta ${ }^{1,3,5,7}$
}

\begin{abstract}
- Additional materials are published online only. To view these files please visit the journal online (http://dx.doi.org/10.1136/ bjophthalmol-2012-301633).

${ }^{1}$ Tissue Engineering and Stem Cell Research Group, Singapore Eye Research Institute, Singapore

${ }^{2}$ School of Chemical and Bimolecular Engineering, National University of Singapore, Singapore ${ }^{3}$ Singapore National Eye Centre, Singapore

${ }^{4}$ School of Mechanical and Aerospace Engineering, Nanyang Technological University, Singapore ${ }^{5}$ Department of Ophthalmology, Yong Loo Lin School of Medicine, National University of Singapore, Singapore ${ }^{6}$ Duke-NUS SRP Neuroscience and Behavioral Disorders, Singapore

${ }^{7}$ Department of Clinical Sciences, Duke-NUS Graduate Medical School, Singapore
\end{abstract}

\section{Correspondence to}

Professor Jodhbir S Mehta,

Singapore Eye Research

Institute, 11 Third Hospital

Drive, Singapore 168751,

Singapore;

jodmehta@gmail.com

Accepted 16 June 2012

\section{UNIOCKED}

This paper is freely available online under the BMJ Journals unlocked scheme, see http:// bjo.bmi.com/site/about/ unlocked.xhtml

\section{ABSTRACT}

Aim Keratoprosthesis (KPro) devices are prone to longterm corrosion and microbiological assault. The authors aimed to compare the inflammatory response and material dissolution properties of two candidate KPro skirt materials, hydroxyapatite $(\mathrm{HA})$ and titania $\left(\mathrm{TiO}_{2}\right)$ in a simulated in vitro cornea inflammation environment.

Methods Lipopolysaccharide-stimulated cytokine secretions were evaluated with human corneal fibroblasts on both $\mathrm{HA}$ and $\mathrm{TiO}_{2}$. Material specimens were subjected to electrochemical and long-term incubation test with artificial tear fluid (ATF) of various acidities. Topography and surface roughness of material discs were analysed by scanning electron microscopy and atomic force microscopy.

Results There were less cytokines secreted from human corneal fibroblasts seeded on $\mathrm{TiO}_{2}$ substrates as compared with $\mathrm{HA}$. $\mathrm{TiO}_{2}$ was more resistant to the corrosion effect caused by acidic ATF in contrast to HA. Moreover, the elemental composition of $\mathrm{TiO}_{2}$ was more stable than HA after long-term incubation with ATF.

Conclusions $\mathrm{TiO}_{2}$ is more resistant to inflammatory degradation and has a higher corrosion resistance as compared with $\mathrm{HA}$, and in this regard may be a suitable material to replace $\mathrm{HA}$ as an osteo-odontokeratoprosthesis skirt. This would reduce resorption rates for KPro surgery.

\section{INTRODUCTION}

Osteo-odonto-keratoprosthesis (OOKP) is a complicated form of ocular surface reconstruction surgery involving removal of a patient's autologous tooth to support an optical cylinder. ${ }^{1-4}$ The OOKP procedure is performed for patients with end-stage ocular surface diseases and severe inflammatory or dry eye states when corneal transplant or other keratoprosthesis (KPro) devices such as the Boston KPro Type I are contraindicated. ${ }^{13}$ However, autologous OOKP surgery cannot be performed when there are no viable canine teeth. Using allograft donor teeth are more prone to lamina resorption, and there are complications related to the use of a bone lamina as a prosthetic skirt including aseptic loosening, bone absorption and limited size of optical field due to the limitations of tooth dimensions. The most severe complication is related to the chronic ocular surface inflammation which can lead to the partial or complete absorption of the bone and tooth implants. ${ }^{5}$ Development of a synthetic substitute for the autologous tooth-bone lamina would therefore be a potential substitute for the OOKP procedure and would also have the added benefit of not requiring the extensive surgery required to remove a canine tooth from the patient.

The ocular surface epithelium not only forms a physical barrier to the external environment but also serves as a non-specific innate immune system against microbes. ${ }^{7}$ In patients with severe ocular surface disease, there is a disruption of the normal innate immunity and a change in the normal commensal flora. ${ }^{9}$ This produces an unstable ocular surface sensitive to the new microbial flora leading to chronic ocular inflammation and these processes are further exacerbated by a poor tear film in these patients. ${ }^{8}$ Therefore any prosthetic device must be able to withstand the unstable ocular surface microenvironment with its continual changing flora and chronic inflammation in these adverse conditions.

It would therefore be advantageous if prospective synthetic KPro skirt devices are able to reduce the incidence of bacterial contamination during implantation as well as maintain excellent tissue biocompatibility in the milieu of an unstable microbial infection. Hydroxyapatite (HA) is a commonly used synthetic biomaterial which has some properties akin to the tooth and bone lamina used in OOKP surgery. ${ }^{10} \mathrm{HA}$ has excellent biocompatibility with human cornea stroma fibroblasts ${ }^{11}$ but is prone to resorption after long-term implantation that can affect the device stability. Biomaterials with improved corrosion resistance at an inflammatory induced microenvironment may offer a more attractive solution. Both acute and chronic inflammatory states are associated with reduced tissue $\mathrm{pH}$ due to the high metabolic activities of inflammatory cells or bacteria. ${ }^{12} 13$ Therefore, the ideal substitute should possess a high corrosion resistance to an inflammatory milieu in an acidic environment. Titania $\left(\mathrm{TiO}_{2}\right)$ has been previously shown to have good biocompatibility with human corneal stroma fibroblasts and epithelial cells. ${ }^{14} 15$ In addition $\mathrm{TiO}_{2}$ has a protective layer of oxide film that is stable, bioinert and provides the material with resistance to corrosion in tissue fluids for example, artificial saliva. ${ }^{16}{ }^{17}$ These properties make $\mathrm{TiO}_{2}$ a potential substitute to $\mathrm{HA}$ as a synthetic OOKP skirt material.

In this study, we aimed to compare the effect of microbial infection on two candidate keratoprosthetic materials, $\mathrm{HA}$ and $\mathrm{TiO}_{2}$. We also aimed to compare the corrosive properties of the materials with an electro-polarisation test and a long-term incubation model. These were performed in artificial tear fluid (ATF) with various acidities to simulate the acidic microenvironment which may occur in a milieu of chronic ocular inflammation. 
Figure 1 Fold change of cytokine expression level at the material surface of lipopolysaccharide (LPS)-stimulated human cornea fibroblasts. All data are relative to the readings of their individual group without LPS treatment. Data are represented as mean $\pm \mathrm{SE}$. ${ }^{*} \mathrm{p}<0.05$ between hydroxyapatite (HA) and (titania) $\mathrm{TiO}_{2}$. bFGF: basic fibroblastic growth factor, G-CSF: granulocyte colony-stimulating factor, GM-CSF: granulocytes macrophage colony-stimulating factor GS: glass slide. $n=6$, IFN: interferon, IL: interleukin, IP: induced protein, MIP: macrophage inflammatory protein, TNF: tumour necrosis factor, VEGF: vascular endothelial growth factor.
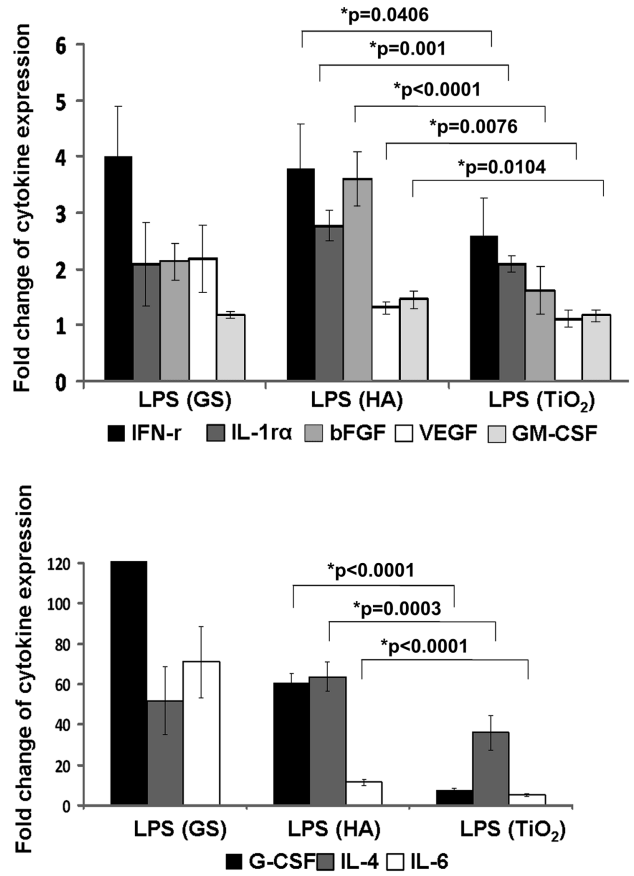
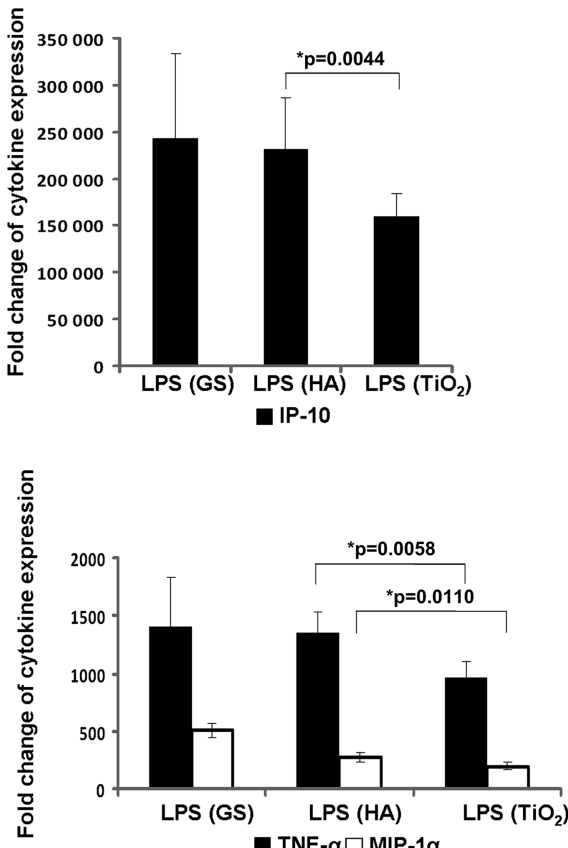

\section{MATERIALS AND METHODS}

\section{Material synthesis}

HA discs were produced by spark plasma sintering HA powder at a nominal pressure of $50 \mathrm{MPa}$ and sintering at $1000^{\circ} \mathrm{C}$. $\mathrm{TiO}_{2}$ discs were produced by spark plasma sintering $\mathrm{TiO}_{2}$ powder at a pressure of $38 \mathrm{MPa}$ and sintering at $1100^{\circ} \mathrm{C}$. The diameters of all discs were $10 \mathrm{~mm}$ and the thicknesses were $2 \mathrm{~mm}$.

\section{Human corneal fibroblasts preparation}

Human corneal stroma was collected from donor corneal tissue. The stroma tissue was cut into small pieces and cultured with the fibroblast growth medium $(10 \%$ fetal bovine serum in Dulbecco's Modified Eagle Medium) changed every 2-3 days. All studies related to human tissues were approved by the Institutional Review Board of the Singapore Eye Research Institute and Singapore National Eye Centre.

\section{Cytokine array}

For cytokine array, corneal stroma fibroblasts $\left(1 \times 10^{4} / \mathrm{cm}^{2}\right)$ in a $30 \mu \mathrm{l}$ droplet were seeded on the surface of the substrate discs $(n=3)$. At $24 \mathrm{~h}$ after seeding, culture medium was changed into serum-free medium containing lipopolysaccharide (LPS, $10 \mathrm{ng}$ /
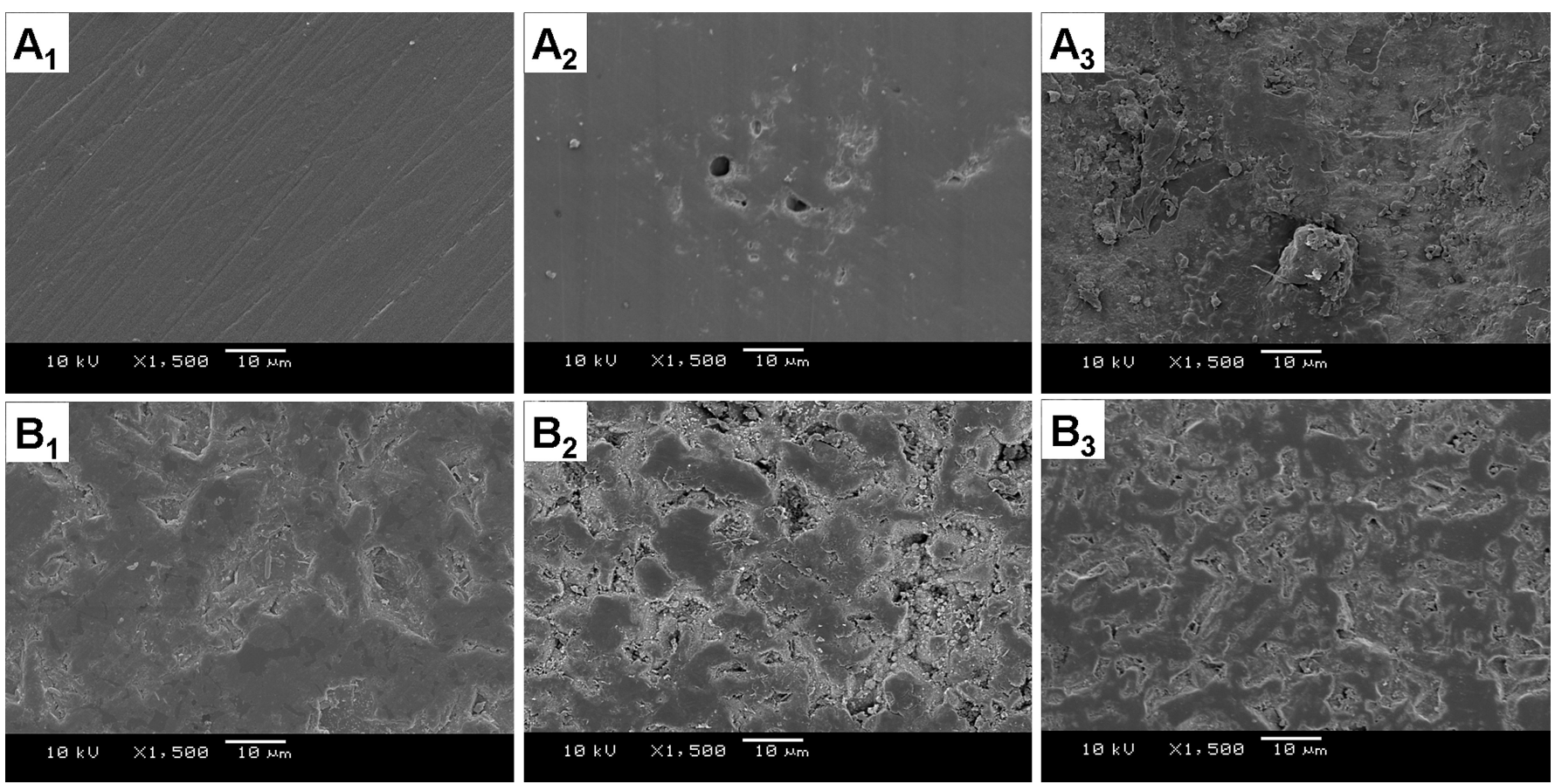

Figure 2 Representative scanning electronic microscopy (SEM) images of hydroxyapatite (HA) and titania $\left(\mathrm{TiO}_{2}\right)$ for electrochemical test. $\mathrm{A}_{1-3}$ : $\mathrm{HA}$; $\mathrm{B}_{1-3}: \mathrm{TiO}_{2} . A_{1}$ and $\mathrm{B}_{1}$ : Before treatment; $A_{2}$ and $\mathrm{B}_{2}$ : After electrochemical test in artificial tear fluid at $\mathrm{pH} 6.5 ; A_{3}$ and $\mathrm{B}_{3}$ : After electrochemical test in artificial tear fluid at $\mathrm{pH}$. The scale bar in all images is $10 \mu \mathrm{m}$. 
ml). Twenty-four hours after addition of LPS, supernatant was collected for Bio-plex Pro Human cytokine 27-plex assay following the standard protocol provided by the vendor (Bio-rad, Berkeley, California, USA). Cytokine analysis of culture medium from cells grown on a glass cover slip was used as a positive control.

\section{Electrochemical test}

Electrochemical tests were carried out in an Autolab-PGSTAT30 (Metrohm Autolab, the Netherlands) at room temperature in a conventional 3-electrode cell. ${ }^{18}$ ATF solution $(0.85 \mathrm{mg} / \mathrm{ml}$ lysozyme, $1.82 \mathrm{mg} / \mathrm{ml}$ bovine serum albumin, $5 \mathrm{mmol} / \mathrm{l}$ urea, $100 \mathrm{mmol} / \mathrm{l} \mathrm{NaCl}, 26 \mathrm{mmol} / \mathrm{l} \quad \mathrm{Na}_{2} \mathrm{HCO}_{3}, 16 \mathrm{mmol} / \mathrm{l} \mathrm{KCl}$, $31 \mu \mathrm{mol} / 1$ citric acid) was made for the immersion of the discs. The $\mathrm{pH}$ was adjusted to 6.5 and 5 by the addition of $\mathrm{HCl}$ solution. ${ }^{19}$ The test was performed with graded sequential incremental potential from -5000 to $5000 \mathrm{mV}$ (saturated calomel electrode) at a scan rate of $10 \mathrm{mV} / \mathrm{s}$.

\section{Long-term corrosion test with simulated tear fluid}

Material discs were incubated in the $50 \mathrm{ml}$ ATF solution $\left(37^{\circ} \mathrm{C}\right)$ continuously for 3 months on a shaker at $60 \mathrm{rpm}$. The solution

\section{A}

$A_{1}$

$\mathrm{A}_{2}$

$\mathrm{A}_{3}$
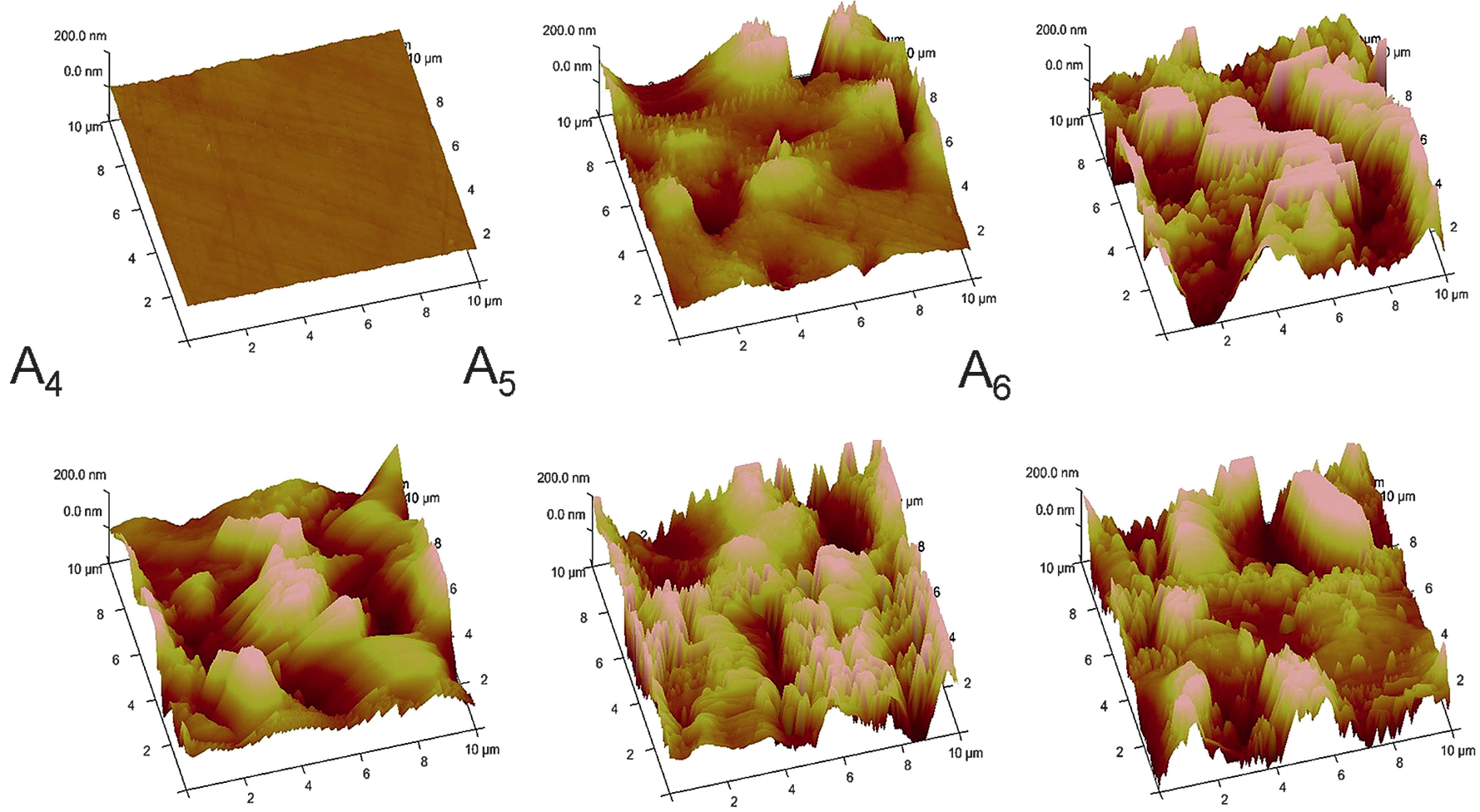

B

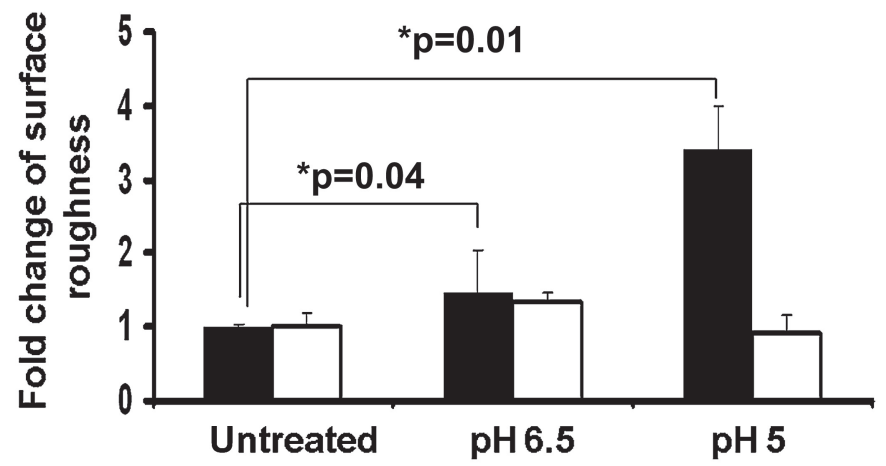

Figure 3 Atomic force microscopy (AFM) scanning and data analysing of hydroxyapatite (HA) and titania $\left(\mathrm{TiO}_{2}\right)$ for electrochemical test. A:

Representative 3D topography of material discs. $A_{1-3}: H A ; A_{4-6}: \mathrm{TiO}_{2} . A_{1}$ and $A_{4}$ : Before treatment; $A_{2}$ and $A_{5}$ : After electrochemical test in artificial tear fluid at $\mathrm{pH} 6.5 ; \mathrm{A}_{3}$ and $\mathrm{A}_{6}$ : After electrochemical test in artificial tear fluid at $\mathrm{pH}$ 5. B: Histogram showing the relationship of average roughness (Ra) with $\mathrm{pH}$ value of artificial tear fluid. All data are normalised to untreated specimens and represented as mean \pm SE. $n=12$. 
was changed once every week. The material discs were then washed in double-distilled water $\left(37^{\circ} \mathrm{C}\right)$ for 1 week with the water changed once every 2 days.

\section{Scanning electronic microscopy (SEM) and atomic force microscopy (AFM)}

The material discs were coated with a layer of gold by the sputter coating machine (BAL-TEC, Germany). Images of the surface of the substrates were scanned with SEM (JEOL 5600LV, Japan). AFM scanning (Veeco, California, USA) was performed with the tapping mode in air (room temperature). The scan rate was $0.5 \mathrm{~Hz} / \mathrm{s}$. To quantify the effect of pitting corrosion, six different areas were randomly selected on each material substrate. Dimensional topography and surface roughness were measured with the software provided by the vendor (Nanoscope $6.11 \mathrm{R} 1$, Veeco, CA, USA). The average roughness ( $R a$ ) of AFM image represents the average pitting corrosion depth. ${ }^{20}$

\section{RESULTS}

\section{Material mediated cellular reaction discrepancy to LPS}

The cytokine expression profile indicated that corneal fibroblasts grown on the different materials continuously released several cytokines: Interleukin (IL)-1r $\alpha$, IL-4, IL-6, IL-9, basic fibroblastic growth factor, granulocyte colony-stimulating factor (G-CSF), granulocytes macrophage colony-stimulating factor (GM-CSF), interferon (IFN)-r, IFN $\gamma$ induced protein-10, macrophage inflammatory protein (MIP)- $1 \alpha$, MIP- $1 b$, regulated on activation normal $T$ cell expressed and secreted, tumour necrosis factor (TNF)- $\alpha$, and vascular endothelial growth factor into the culture medium, and the secretions were increased following LPS stimulation. After normalisation of the cytokine expression level to the control, the data showed that there were less cytokines (IFN-g, IL-1r $\alpha$, basic fibroblastic growth factor, vascular endothelial growth factor, GM-CSF, induced protein -10, G-CSF, IL-4,
IL-6, TNF- $\alpha$, MIP-1 $\alpha$ ) stimulated by LPS from corneal fibroblasts on $\mathrm{TiO}_{2}$ substrates than $\mathrm{HA}$ (figure 1).

\section{Assessment of surface corrosion after electrochemical test}

Untreated HA whose topography was qualitatively assessed by SEM (figure 2A1) exhibited a smooth and pit-free surface. After electro-polarisation testing with ATF ( $\mathrm{pH}$ 6.5), clusters of deep pits were found on the surface of HA discs (figure 2A2). After testing with acidic ATF ( $\mathrm{pH} 5$ ), the surface of the HA disc was seen to be unevenly damaged with HA particles exposed to the air (figure 2A3). SEM images of untreated $\mathrm{TiO}_{2}$ exhibited a surface of uneven ridges (figure 2B1). After electro-polarisation in ATF ( $\mathrm{pH}$ 6.5), there were some shallow pits on the surface of $\mathrm{TiO}_{2}$ that were less in nature and not as clearly defined as those on HA (figure 2B2). After electro-polarisation in $\mathrm{pH} 5 \mathrm{ATF}$, there was no qualitative increase in the amount of pits on the surface of $\mathrm{TiO}_{2}$ (figure 2B3).

The depths of pits, cleavages and the overall corrosion severity were quantitatively assessed by the 3D topography of AFM Representative topography was captured from each substrate after electro-polarisation in ATF. No obvious pits could be observed on the normal HA samples (figure 3A1). The material surface became uneven after electrochemical testing with ATF ( $\mathrm{pH}$ 6.5, figure 3A2). The surface roughness significantly increased on the HA discs after electro-polarisation with acidic ATF ( $\mathrm{pH}=5$, figure 3A3). The 3D topography of $\mathrm{TiO}_{2}$ showed no obvious change of surface roughness after ATF treatment at either $\mathrm{pH}$ (figure 3A4-A6). Following the capture of topography with each tear fluid, the mean depth of the surface roughness (Ra) was calculated. $R a$ of untreated HA was $23.1 \pm 1.1 \mathrm{~nm}$. After electro-polarisation with $\mathrm{pH} 6.5 \mathrm{ATF}$, the $\mathrm{Ra}$ increased to $34.1 \pm 13.4 \mathrm{~nm}$. After electro-polarisation with pH 5 ATF, $R a$ of HA specimens increased around twofold and reached as high as $78.7 \pm 13.2 \mathrm{~nm}$. The $\mathrm{Ra}$ of untreated $\mathrm{TiO}_{2}$ specimen was $90.8 \pm 16.8 \mathrm{~nm}$ and showed no significant change after electro-
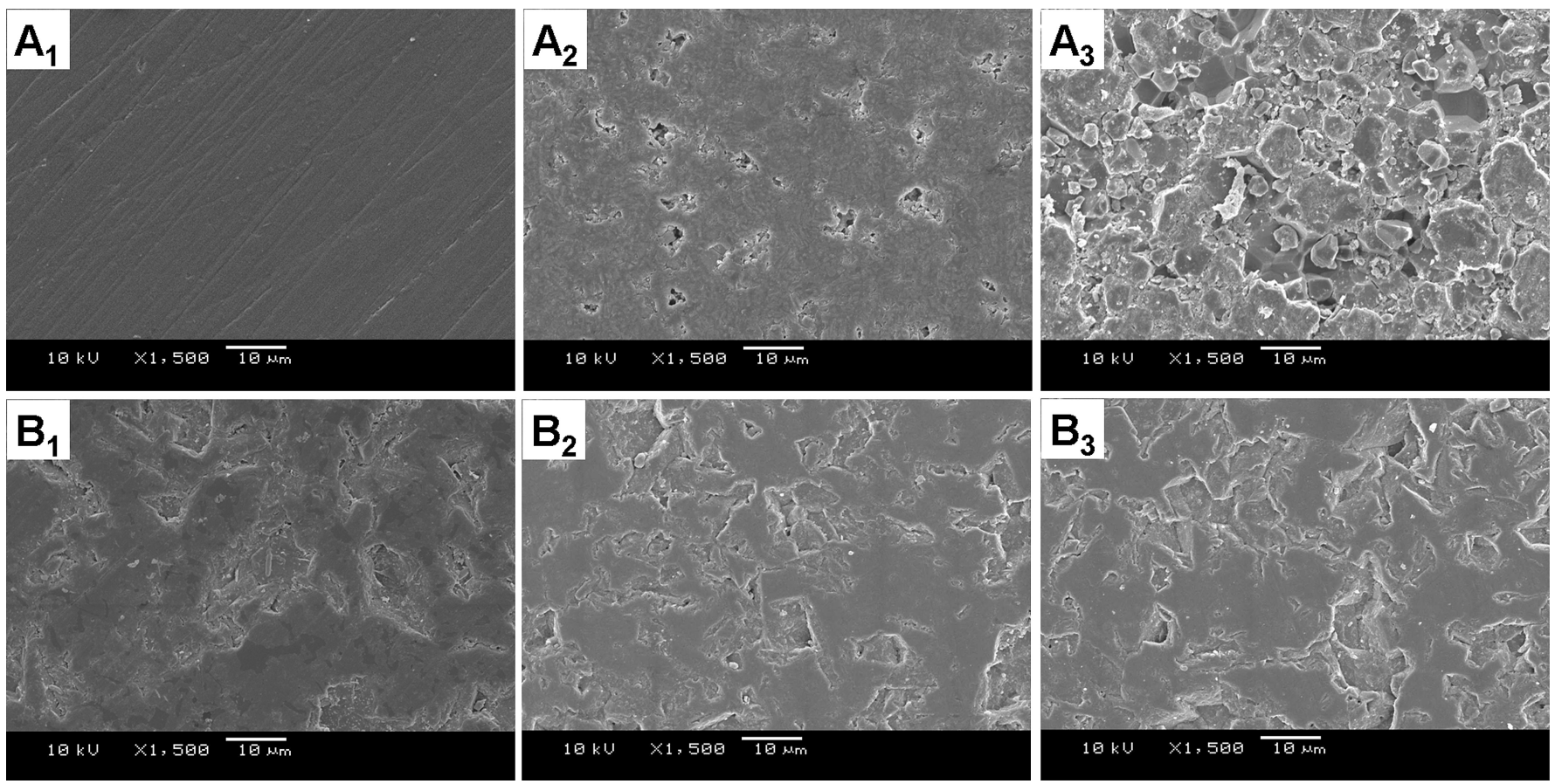

Figure 4 Representative scanning electronic microscopy (SEM) images of hydroxyapatite (HA) and titania $\left(\mathrm{TiO}_{2}\right)$ for long-term corrosion test. $\mathrm{A}_{1-3}$ : $H A ; B_{1-3}: T_{i O}$. $A_{1}$ and $B_{1}$ : Before treatment; $A_{2}$ and $B_{2}$ : After long-term incubation in artificial tear fluid at pH 6.5; $A_{3}$ and $B_{3}$ : After long-term incubation in artificial tear fluid at pH 5 . The scale bar in all images is $10 \mu \mathrm{m}$. 
polarisation with pH 6.5 ATF or pH 5 ATF $(R a=121.8 \pm 11.4 \mathrm{~nm}$ and $83.3 \pm 20.8 \mathrm{~nm}$ respectively, $\mathrm{p}>0.05)$. After normalisation to the untreated $\mathrm{HA}$ or $\mathrm{TiO}_{2}$, the fold changes of $\mathrm{Ra}$ were plotted in figure 4C.

\section{Assessment of surface corrosion after long-term incubation with \\ ATF}

After incubation with $\mathrm{pH} 6.5$ ATF for 3 months, there was a high density of deep pits developed on the surface of HA (figure 4A2). After incubation with $\mathrm{pH} 5 \mathrm{ATF}$, there was severe disruption of the HA surface. Deep corrosion pits and cracks merged together with multiple HA particles being exposed on the surface (figure 4A3). For $\mathrm{TiO}_{2}$ specimens, there was no obvious surface morphology change on SEM images after longterm incubation with ATF ( $\mathrm{pH} 6.5$ or $\mathrm{pH}$ 5) (figure 4B1-3).

The surface topography of material discs after long-term degradation test was also quantitatively assessed by AFM. The 3D topography of material discs after incubation with $\mathrm{pH} 6.5$ ATF revealed that HA surface became very uneven (figure 5A2). The HA surface was extremely rough after incubation with $\mathrm{pH} 5$

A

$A_{1}$

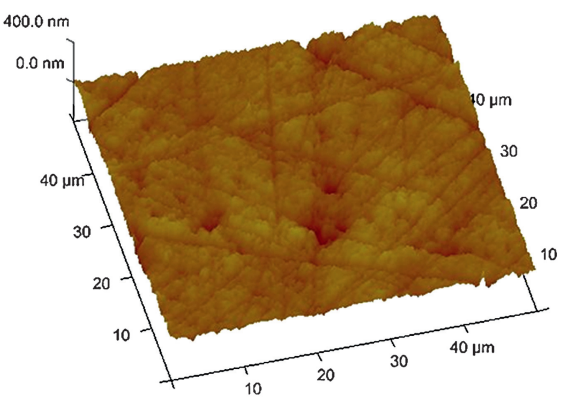

$\mathrm{A}_{4}$

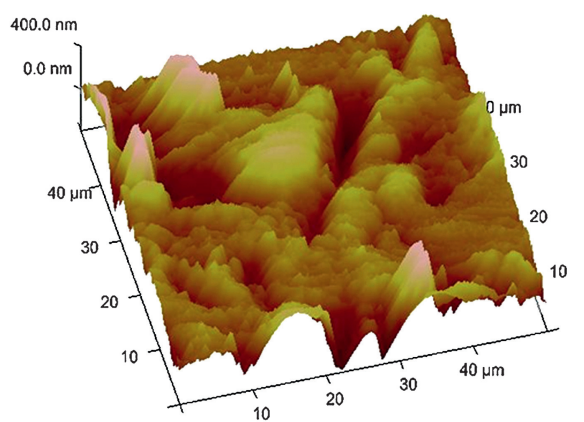

$\mathrm{A}_{2}$

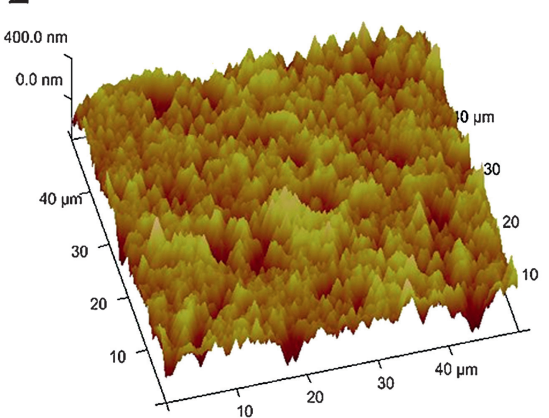

$\mathrm{A}_{5}$

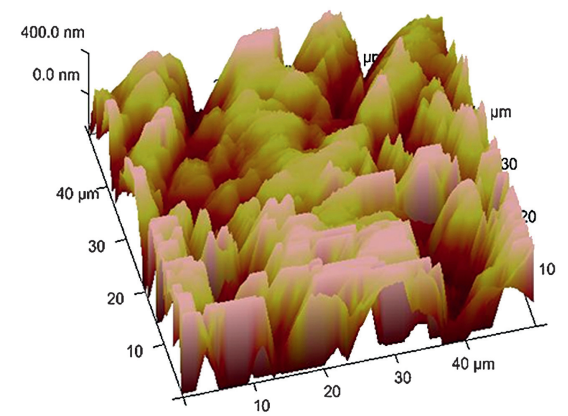

$\mathrm{A}_{3}$

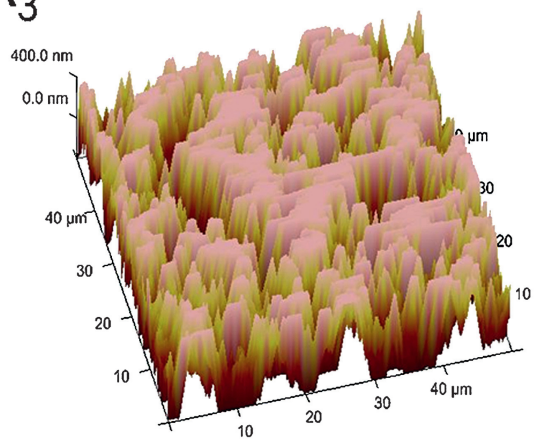

$A_{6}$

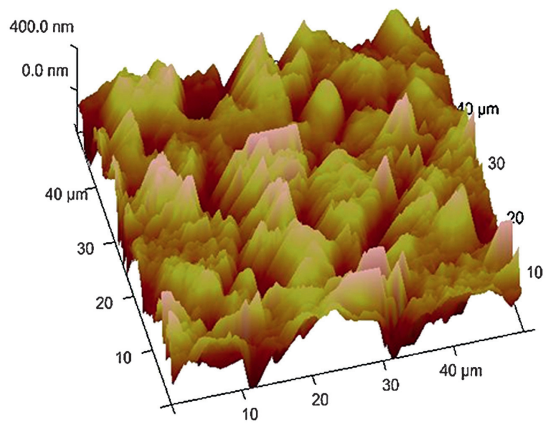

B

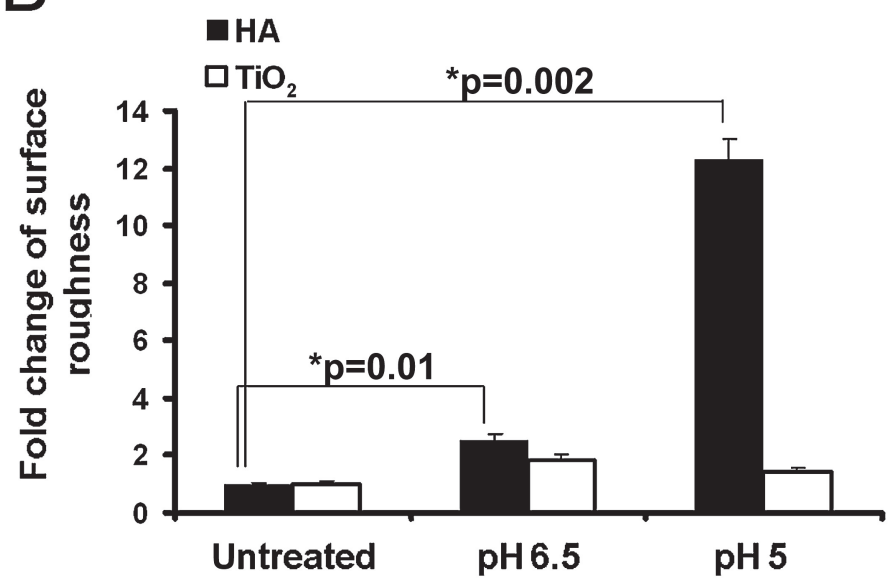

Figure 5 Atomic force microscopy (AFM) scanning and data analysing of hydroxyapatite (HA) and titania ( $\left.\mathrm{TiO}_{2}\right)$ for long-term corrosion test. A: Representative 3D topography of material discs. $A_{1-3}: H A ; A_{4-6}: T_{0} O_{2} A_{1}$ and $A_{4}$ : Before treatment; $A_{2}$ and $A_{5}$ : After long-term corrosion test in artificial tear fluid at $\mathrm{pH}$ 6.5; $\mathrm{A}_{3}$ and $\mathrm{A}_{6}$ : After long-term corrosion test in artificial tear fluid at $\mathrm{pH}$ 5. B: Histogram showing the relationship of average roughness $(R a)$ with $\mathrm{pH}$ value of artificial tear fluid. All data are normalised to untreated specimens and represented as mean \pm SE. $n=12$. 
ATF (figure 5A3). The 3D topography of $\mathrm{TiO}_{2}$ showed a nonsignificant increase of surface roughness after ATF incubation (figure 5A4-6).Surface $R a$ of untreated HA was $24.1 \pm 1.1 \mathrm{~nm}$. After treatment with pH 6.5 ATF, the $R a$ significantly increased around twofold to $62.2 \pm 3.4 \mathrm{~nm}$. The $R a$ of HA specimen significantly increased 12-fold and reached as high as $296 \pm 12.2 \mathrm{~nm}$ in pH 5 ATF. The $\mathrm{Ra}$ of $\mathrm{TiO}_{2}$ specimen increased from $93.1 \pm 8.3$ to $152.4 \pm 0.2 \mathrm{~nm}$ after incubation with $\mathrm{pH} 6.5 \mathrm{ATF}$ and increased to $135 \pm 10.7 \mathrm{~nm}$ after treatment with $\mathrm{pH} 5$ ATF. Both these increases were less than onefold compared to the untreated $\mathrm{TiO}_{2}$. The image depth readings were normalised to the untreated $\mathrm{HA}$ or $\mathrm{TiO}_{2}$ samples and the fold changes of $R a$ were plotted in figure $5 \mathrm{C}$.

\section{DISCUSSION}

There are currently few studies evaluating the dissolution properties of prospective artificial corneal skirt devices. ${ }^{21} 22$ One of the issues in OOKP surgery is resorption. This can lead to leakage around the optic cylinder which in turn can predispose to microbiological infection and the risk of the resorbed lamina harbouring organisms. In our designed synthetic OOKP device, the optic will be sealed to the haptic with the use of dental cement as in OOKP, thereby reducing the possibilities of an intraocular infection. Ideally our study would have a comparative group consisting of osteodental lamina, even though synthetic HA and osteodental lamina are not totally comparable in all respects. The mineral constituents of synthetic HA and osteodental lamina are comparable. Both of them are made of calcium apatite with the formula $\mathrm{Ca}(\mathrm{n})(\mathrm{PO} 4)(\mathrm{n})(\mathrm{OH})(\mathrm{n})$. Hence they share some similar physical and chemical properties. That is why we and others ${ }^{21}$ have used HA as control group. Comparison studies with human cadaver osteodental lamina would have been more informative but currently we have no such resources and it will be our future work.

We analysed the inflammatory cellular response and dissolution properties of $\mathrm{HA}$ and $\mathrm{TiO}_{2}$ in a simulated inhospitable ocular surface environment. LPS is a component of the cell membrane of gram-negative bacteria. Corneal fibroblasts are sensitive to LPS stimulation and this plays a role in the regulation of cornea inflammation secondary to bacterial infection. LPS has been shown to induce the release of the pro-inflammatory cytokine IL6 and chemokines G-CSF, monocyte chemotactic protein-1, MIP-1 $\beta$, IL-8 and intercellular adhesion molecule- 1 by corneal fibroblasts. ${ }^{23} 24$ Likewise infectious corneal ulcers are associated with the production of pro-inflammatory cytokines, such as IL-1, TNF- $\alpha$ and chemokines such as GM-CSF by resident human corneal fibroblasts. ${ }^{25-27}$ Such a response in vivo would trigger local infiltration and activation of leucocytes and contribute to localised inflammation. In our studies, we found that LPS induced the production of a wide panel of cytokines/chemokines from cultured human corneal fibroblasts. We found that HA-grown keratocytes were more prone to LPS stimulated cytokines/ chemokines secretion than $\mathrm{TiO}_{2}$ (figure 1). The reasons that human cornea fibroblasts on $\mathrm{TiO}_{2}$ substrates secreted less cytokine/chemokines than those from HA are not fully understood, but could simply represent the fact that $\mathrm{TiO}_{2}$ presents a more bioinert surface as compared to HA. The difference in cytokine expression we detected was due to the intrinsic properties of the material substrates on the cells. 3-(4,5-Dimethylthiazol-2-yl)-2,5diphenyltetrazolium bromide (MTT) assay excluded the possibility that this difference was due to cellular toxicity caused by LPS on the fibroblasts grown on either biomaterial substrates (supplementary figure). Our results suggest that $\mathrm{TiO}_{2}$ fibroblasts are less responsive to LPS stimulated inflammation and hence may prove effective in reducing the propagation of an acute inflammatory response into a chronic one.

Following ischaemia and inflammation, there is a reduction in tissue $\mathrm{pH} .{ }^{12}{ }^{13}$ The reported reduction in $\mathrm{pH}$ can vary from 0.1 to $2.0 .^{28}$ Therefore, to address the issue of implant device stability in an ocular inflammatory milieu, we compared the corrosion resistance properties of material specimens in ATF at a $\mathrm{pH}$ value of 6.5 and also at a more acidic $\mathrm{pH}(\mathrm{pH} 5)$ to simulate an inhospitable ocular surface. We compared the corrosion resistance properties of $\mathrm{TiO}_{2}$ and $\mathrm{HA}$ in an electric-polarisation facility. Bioactive HA has a low polarisability and electric conductivity hence it is not possible to compare the electrochemical index of $\mathrm{HA}$ directly with $\mathrm{TiO}_{2}$. Morphological changes after electrochemical treatment were analysed, and compared with $\mathrm{HA}$. $\mathrm{TiO}_{2}$ showed less degradation with electrochemical treatment at both $\mathrm{pH} 6.5$ and $\mathrm{pH} 5$ ATF (figures 2 and 3 ). We deduce that this may be due to the passive film formation at the interface between $\mathrm{TiO}_{2}$ and ATF.

Our SEM and AFM data showed that in pH 6.5 ATF, both HA and $\mathrm{TiO}_{2}$ developed some early signs of pitting corrosion formation and an increase in surface roughness (figure 2 to figure 5). However, the signs of corrosion in $\mathrm{pH} 5$ ATF were remarkably different as $\mathrm{TiO}_{2}$ showed smaller pits and a gradual increase in surface roughness while HA showed large pit formations and a steep increase in surface roughness (figure 2 to figure 5). These observations indicated that, in a milieu of an acidic ocular tissue, $\mathrm{TiO}_{2}$ is more resistant to corrosion as compared with HA.

Chronic inflammation plays an essential role in OOKP lamina degradation. ${ }^{5}$ Hence, in our study we compared the corrosion resistance properties of $\mathrm{HA}$ and $\mathrm{TiO}_{2}$ after long-term incubation with low $\mathrm{pH}$ ATF which stimulated a chronic ocular inflammation state. $\mathrm{TiO}_{2}$ showed no significant degradation of surface morphology change while the surface of $\mathrm{HA}$ was severely damaged in both $\mathrm{pH} 6.5$ and $\mathrm{pH} 5$ ATF (figure 4 and figure 5). The analysis of the elemental compositions showed that ' $\mathrm{Ca}$ ' and ' $\mathrm{P}$ ' were continuously released from HA while in contrast the elemental composition of $\mathrm{TiO}_{2}$ was stable (Supplementary table). Therefore, our data again indicated that $\mathrm{TiO}_{2}$ is more stable to long-term corrosion. $\mathrm{TiO}_{2}$ might not undergo significant resorption in an adverse milieu and thereby may not lead to a leak in the KPro skirt area around the optical cylinder.

In conclusion, our studies involving cytokine array to compare the material mediated cellular reaction to inflammatory stimuli and studies evaluating the morphological changes of material substrates induced by acidic ATF show that $\mathrm{TiO}_{2}$ is a promising skirt material for a fully synthetic OOKP device in terms of corrosion resistance to microbial infection and device stability.

Acknowledgements The authors acknowledge funding (HREF/R684) from the Singapore Eye Research Institute and Shaw Foundation, Singapore.

Contributors (1) Substantial contributions to conception and design, acquisition of data, or analysis and interpretation of data: XWT, AR, ZLS, ACST, JSM. (2) Drafting the article or revising it critically for important intellectual content; XWT, KGN, DT, KAK, RWB, JSM. (3) Final approval of the version to be published: JSM.

Funding This work was supported by Singapore Shaw's Foundation.

\section{Competing interests None.}

Provenance and peer review Not commissioned; externally peer reviewed.

\section{REFERENCES}

1. Liu C, Paul B, Tandon R, et al. The osteo-odonto-keratoprosthesis (OOKP). Semin Ophthalmol 2005;20:113-28.

2. Tan DT, Tay AB, Theng JT, et al. Keratoprosthesis surgery for end-stage corneal blindness in Asian eyes. Ophthalmology 2008;115:503-10.e3. 
3. Tay AB, Tan DT, Lye KW, et al. Osteo-odonto-keratoprosthesis surgery: a combined ocular-oral procedure for ocular blindness. Int J Oral Maxillofac Surg 2007;36:807-13.

4. Falcinelli G, Falsini B, Taloni M, et al. Modified osteo-odonto-keratoprosthesis for treatment of corneal blindness: long-term anatomical and functional outcomes in 181 cases. Arch Ophthalmol 2005:123:1319-29.

5. Hille K, Landau $\mathrm{H}$, Ruprecht KW. Osteo-odonto-keratoprosthesis. A summary of 6 years surgical experience. Ophthalmologe 2002;99:90-5.

6. Iyer G, Pillai VS, Srinivasan B, et al. Modified osteo-odonto keratoprosthesis-the Indian experience-results of the first 50 cases. Cornea 2010;29:771-6.

7. Akpek EK, Gottsch JD. Immune defense at the ocular surface. Eye (Lond) 2003:17:949-56.

8. Ueta M, Kinoshita S. Ocular surface inflammation mediated by innate immunity. Eye Contact Lens 2010;36:269-81.

9. Ueta M, Kinoshita S. Innate immunity of the ocular surface. Brain Res Bull 2010;81:219-28.

10. Polack FM, Heimke G. Ceramic keratoprostheses. Ophthalmology 1980;87:693-8.

11. Mehta JS, Futter CE, Sandeman SR, et al. Hydroxyapatite promotes superior keratocyte adhesion and proliferation in comparison with current keratoprosthesis skirt materials. Br J Ophthalmol 2005;89:1356-62.

12. Pullamsetti SS, Savai R, Janssen W, et al. Inflammation, immunological reaction and role of infection in pulmonary hypertension. Clin Microbiol Infect 2011:17:7-14.

13. Punnia-Moorthy A. Evaluation of $\mathrm{pH}$ changes in inflammation of the subcutaneous air pouch lining in the rat, induced by carrageenan, dextran and Staphylococcus aureus. J Oral Pathol 1987;16:36-44.

14. Tan XW, Perera AP, Tan A, et al. Comparison of candidate materials for a synthetic osteo-odonto keratoprosthesis device. Invest Ophthalmol Vis Sci 2011;52:21-9.

15. Ament JD, Spurr-Michaud SJ, Dohlman CH, et al. The Boston Keratoprosthesis: comparing corneal epithelial cell compatibility with titanium and PMMA. Cornea 2009;28:808-11.

16. Anwar EM, Kheiralla LS, Tammam RH. Effect of fluoride on the corrosion behavior of Ti and Ti6Al4V dental implants coupled with different superstructures. J Oral Implantol 2011;37:309-17.
17. Yu W0, Qiu J, Zhang FO. In vitro corrosion study of different TiO2 nanotube layers on titanium in solution with serum proteins. Colloids Surf B Biointerfaces 2011:84:400-5.

18. Huang HH. Surface characterizations and corrosion resistance of nickel-titanium orthodontic archwires in artificial saliva of various degrees of acidity. J Biomed Mater Res A 2005:74:629-39.

19. Alexeev VL, Das S, Finegold DN, et al. Photonic crystal glucose-sensing material for noninvasive monitoring of glucose in tear fluid. Clin Chem 2004:50:2353-60.

20. Yuan SJ, Pehkonen SO. Microbiologically influenced corrosion of 304 stainless steel by aerobic Pseudomonas NCIMB 2021 bacteria: AFM and XPS study. Colloids Surf B Biointerfaces 2007:59:87-99.

21. Viitala R, Franklin V, Green D, et al. Towards a synthetic osteo-odontokeratoprosthesis. Acta Biomater 2009;5:438-52.

22. Laattala $\mathbf{K}$, Huhtinen R, Puska $\mathrm{M}$, et al. Bioactive composite for keratoprosthesis skirt. J Mech Behav Biomed Mater 2011;4:1700-8.

23. Bazan HE. Cellular and molecular events in corneal wound healing: significance of lipid signalling. Exp Eye Res 2005;80:453-63.

24. Fukuda K, Kumagai N, Fujitsu Y, et al. Fibroblasts as local immune modulators in ocular allergic disease. Allergol Int 2006;55:121-9.

25. Cubitt CL, Lausch RN, Oakes JE. Differential regulation of granulocyte macrophage colony-stimulating factor gene expression in human corneal cells by pro-inflammatory cytokines. J Immunol 1994;153:232-40.

26. Hong JW, Liu JJ, Lee JS, et al. Proinflammatory chemokine induction in keratocytes and inflammatory cell infiltration into the cornea. Invest Ophthalmol Vis Sci 2001:42:2795-803

27. Thakur A, Willcox MD. Cytokine and lipid inflammatory mediator profile of human tears during contact lens associated inflammatory diseases. Exp Eye Res 1998:67:9-19

28. Andersson SE, Lexmuller $\mathrm{K}$, Johansson $\mathrm{A}$, et al. Tissue and intracellular $\mathrm{pH}$ in normal periarticular soft tissue and during different phases of antigen induced arthritis in the rat. J Rheumatol 1999;26:2018-24. 